\title{
Obstructive sleep apnoea caused by a pleomorphic adenoma: a case report
}

\begin{abstract}
Background: Sleep apnoea syndrome is a condition characterised by excessive daytime somnolence, hypnagogic hallucinations, personality and sexual behaviour changes and hypertension. Most commonly, sleep apnoea syndrome is secondary to upper airway obstruction as a result of intermittent hypotonia of the soft palate and tongue musculature. In rare occasions, obstructive sleep apnoea can result from anatomical abnormalities such as a mass in the upper aerodigestive tract. ${ }^{1}$
\end{abstract}

Case summary: A 40 year old gentleman was referred in view of obstructive sleep apnoea symptoms. On examination, a large right sided lesion was seen in the oropharynx. Further investigations with flexible nasoendoscopy, computed tomography $(\mathrm{CT})$ and magnetic resonance imaging (MRI) of the neck were carried out which confirmed a large mass most likely arising from the right tonsillar bed. Surgical excision was carried out via a transoral approach. Histology was reported as pleomorphic adenoma.

Conclusion: It is widely accepted that management of obstructive sleep apnoea involves treating the cause together with conservative and lifestyle measures. The majority of patients do not require surgery but a thorough assessment, including that of the upper aerodigestive tract, should be carried out to exclude rare causes of obstructive sleep apnoea. ${ }^{2}$

Keywords: Obstructive sleep apnoea, pleomorphic adenoma
Volume 10 Issue 2 - 2018

\author{
Marija Agius Spiteri,' Gabriella Grech,' \\ Amanda Bartolo,' Sinisa Lalicevic,' Eric John \\ Farrugia' \\ 'Otorhinolaryngology and Head \& Neck Surgery Department, \\ Mater Dei Hospital, Malta \\ ${ }^{2}$ Department of Sugery, Mater Dei Hospital, Malta
}

Correspondence: Marija Agius Spiteri, Otorhinolaryngology and Head \& Neck Surgery Department, Mater Dei Hospital, 47, Hompesch Road Fgura, Malta, Tel +356992456I3, Email marija.agius@gmail.com

Received: February 06, 2018 | Published: March 07, 2018
Abbreviations: CT, Computed tomography; MRI, Magnetic resonance imaging; OSA, Obstructive sleep apnoea

\section{Literature review}

Obstructive sleep apnoea is a condition involving intermittent collapse of the pharynx, usually at the level of soft palate, causing episodes of apnoea during sleep ${ }^{1}$. Muscle tone declines during sleep and thus, upper airway dilating muscles are unable to maintain pharyngeal patency. In severe cases, the patient wakes up briefly multiple times during the night to allow dilating muscles to re-open the airway ${ }^{2}$. This results in severe sleep fragmentation leading to symptoms such as daytime somnolence (assessed by Epworth sleepiness scale), morning headaches and decreased cognitive performance. Polysomnography is the gold standard investigation to diagnose obstructive sleep apnoea. This involves monitoring of oxygen saturation, airflow at the nose and mouth, ECG, EMG, chest and abdominal movement during sleep ${ }^{1}$. The occurrence of 15 or more episodes of apnoea or hypopnoea during 1 hour of sleep indicates significant sleep apnoea. ${ }^{3}$

Pleomorphic adenoma is a benign salivary gland tumour known for its pleomorphic appearance under light microscopy. It is the most common tumour of the salivary glands with $80-90 \%$ which arise from the parotid gland. ${ }^{4}$ Spiro in his study of 2078 patients with salivary gland neoplasia reported that $20-40 \%$ of all salivary gland tumours arise from minor salivary glands, mostly from the palate (42.63\%), lip $(10 \%)$, buccal mucosa $(5.5 \%)$, retromolar area $(0.7 \%)$ as well as floor of the mouth, tongue, tonsil, pharynx and nasal cavity. ${ }^{5-9}$

In rare occasions, obstructive sleep apnoea is a result of anatomical abnormalities such as a mass in the upper aerodigestive tract. Apart from obstructive sleep apnoea, tumours of this location may also cause dyspnoea, dysphagia and airway obstruction. ${ }^{10}$ The first case reported, was in 1981 where a patient was diagnosed with obstructive sleep apnoea secondary to nasopharyngeal carcinoma ${ }^{3}$. Up to 2005 only 30 cases of obstructive sleep apnoea caused by head and neck tumours have been reported. ${ }^{11}$ In the English language literature, 9 pleomorphic adenomas were found to cause OSA. ${ }^{12}$

Pleomorphic adenomata are more common in patients between 40 to 60 years of age with a male to female ratio of $2: 3^{4}$. However case reports involving a 7 year old patient and an 82 year old patient are also seen. ${ }^{13}$ Such tumours are usually asymptomatic and thus they can grow a long time before being diagnosed. The potential risk of malignant transformation increases over the years with an incidence of 1 to $7 \% .{ }^{14}$ The smaller the salivary gland that is affected, the more likely it is to trigger a malignant tumour. ${ }^{10}$

The diagnostic work-up begins with haematologic and serologic tests followed by imaging ${ }^{6}$. MRI provides precise information about tumour margins as well as the relationship with the surrounding structures. Fine Needle Aspiration Cytology (FNAC) can also be used to aid with cytological diagnosis. There are different approaches that can be used in pleomorphic adenomata of the palate. It is of utmost importance to have adequate exposure to identify and protect vital structures and ensure complete removal. Intra-oral and extra-oral interventions can be used. Transparotid, transcervical and transpharyngeal approaches are the extra-oral approaches which are commonly used. Mandibulotomy can be performed to improve exposure. ${ }^{4}$

\section{Case summary}

A 42 year old male, with no previous medical history was referred to the Otorhinolaryngology and Head \& Neck surgery outpatients' 
department at Mater Dei Hospital with Obstructive Sleep Apnoea (OSA) symptoms. The patient admitted to a few year history of such symptoms. During examination, a large right sided lesion was seen in the oropharynx which seemed to be arising from the soft palate. This lesion was seen to be crossing the midline and most likely contributing to his obstructive symptoms. The patient underwent a flexible nasoendoscopy which showed a large lesion extending to the lateral nasal cavity. Subsequently the patient underwent urgent imaging with computed tomography (CT) (Figure 1) of the neck and thorax which showed a large mass with the most likely site of origin being in the right tonsillar bed. A dedicated magnetic resonance imaging (MRI) (Figure 2) (Figure 3) of the neck was recommended for staging, to better assess the extent of the lesion. Two hypoechoic intrahepatic liver lesions were were also noted. A biopsy of the lesion arising from soft palate was taken under local anaesthesia which was reported as adenomatoid acinar hyperplasia.

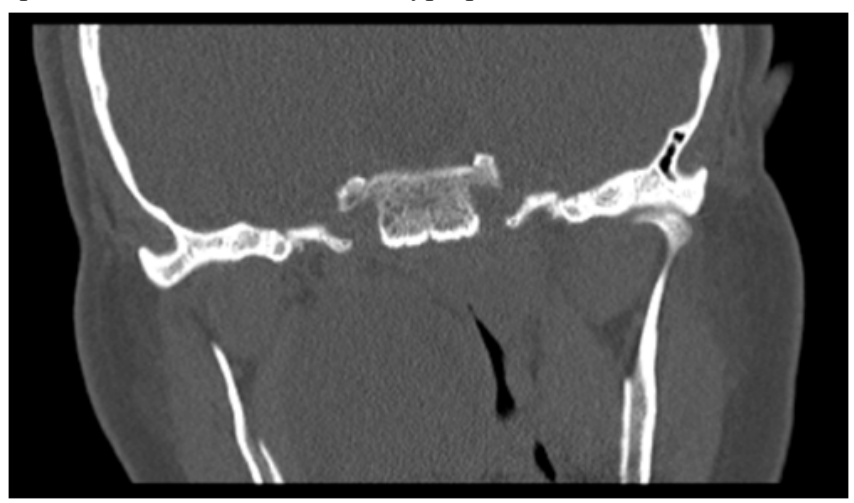

Figure I Coronal CT scan showing a large right sided soft tissue swelling causing almost complete obstruction of the upper airway.

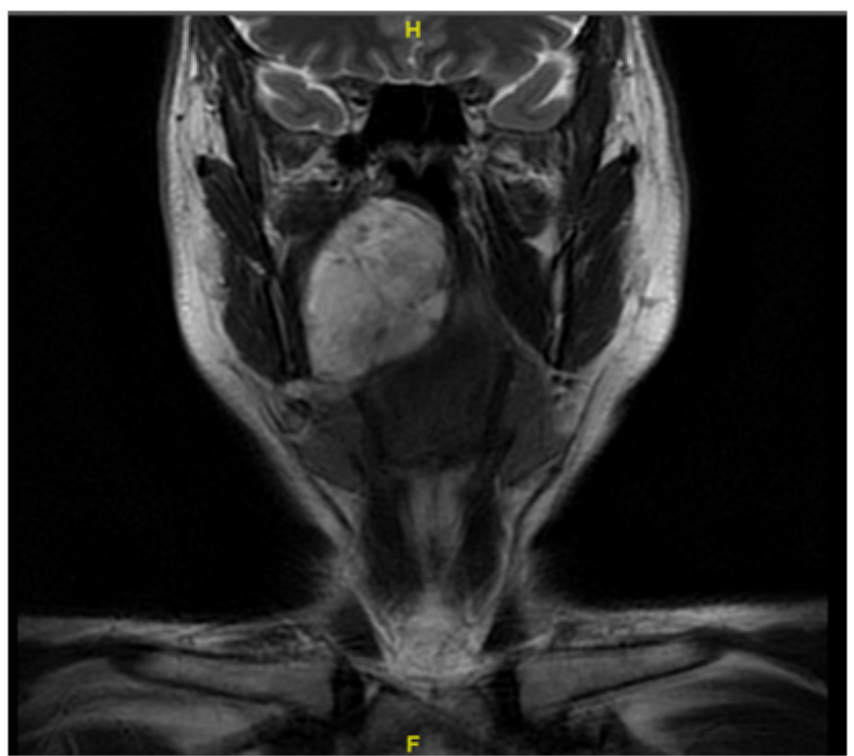

Figure 2 Coronal MR scan showing a large right sided parapharyngeal lesion causing incomplete upper airway obstruction.

The patient underwent a MRI of the liver and spleen which showed signs of iron overload and reported the liver lesions as benign haemangiomas. Long discussions were undertaken with the patient and his wife with the proposed surgery with particular focus on the eventuality of tracheostomy as well as open approach.

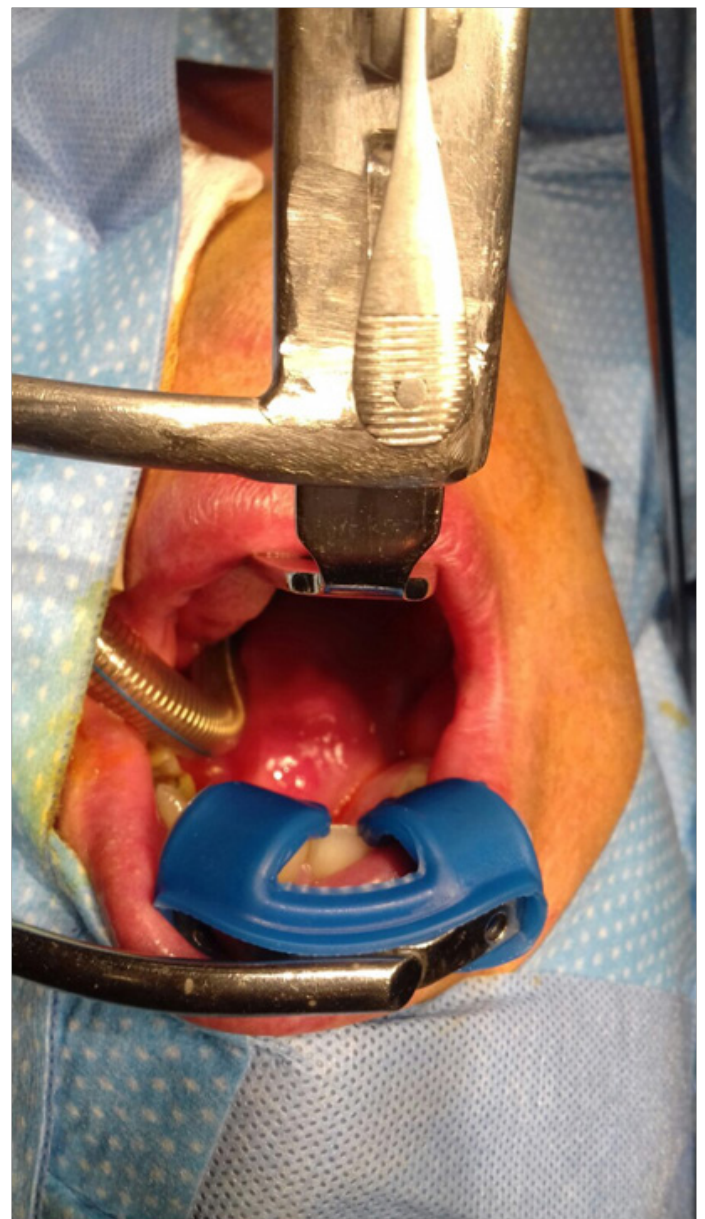

Figure 3 Intra-operative photograph at the start of surgery with large palatal lesion.

A transoral approach was used using a Boyle Davies gag and cheek retractors to aid with access and exposure. Cheek retractors proved to be handy for better exposure. The lesion was 4 x $5 \mathrm{~cm}$ in diameter, partly cystic and unfortunately the capsule was breached during the manipulation of the tumour. After resection, the defect was closed primarily with absorbable sutures in an interrupted fashion (Figure 4) (Figure 5).

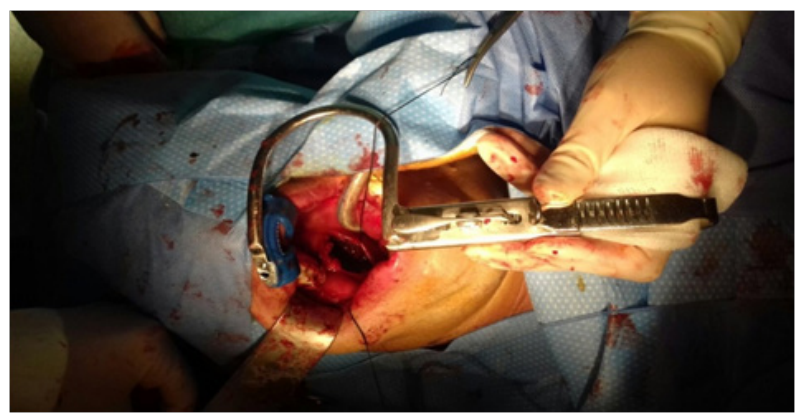

Figure 4 Intra operative photographs showing the defect after the excision of the tumour. 


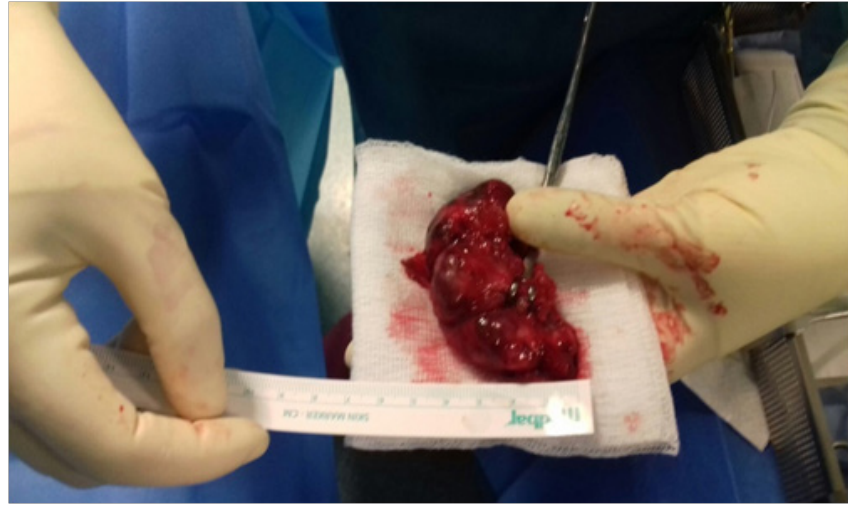

Figure 5 Specimen dimensions $4 \times 5 \mathrm{~cm}$.

The patient was sent to Intensive Care Unit for overnight monitoring and was discharged on the second postoperative day. The final histology was reported as pleomorphic adenoma.

The patient is being followed up regularly at the outpatients' clinic.

\section{Conflict of interest}

None declared.

\section{References}

1. Longmore JM. Oxford handbook of clinical medicine. Oxford University Press. 2010; p. 194.

2. Colledge NR, Walker BR, Ralston S, et al. Davidson's principles and practice of medicine. Edinburgh: Churchill Livingstone/Elsevier. 2010; pp. 1440.

3. Moses C, Buscemi L. Obstructive Sleep Apnea Syndrome Associated with Nasopharyn-geal Carcinoma. West J Med. 1981;134(1):69-70.
4. Datarkar A, Deshpande A. Giant Parapharyngeal Space Pleomorphic Adenoma of the Deep Lobe of Parotid Presenting as Obstructive Sleep Apnoea: A Case Report \& Review of the Diagnostic and Therapeutic Approaches. Journal of Maxillofacial and Oral Surgery. 2014;14(3):532-537.

5. Spiro RH. Salivary neoplasms: overview of a 35-year experience with 2,807 patients. Head Neck Surg. 1986;8(3):177-184.

6. Akın I, Karagöz T, Mutlu M, et al. Pleomorphic Adenomas of the Parapharyngeal Space. Case Reports in Otolaryngology. 2014;2014:14.

7. Sharma N, Singh V, Malhotra D, et al. Pleomorphic Adenoma of the hard palate- A case report. Indian journal of dental sciences. 2010;2(1):18-20.

8. Gothwal AK, Kamath A, Pavaskar RS, et al. Pleomorphic Adenoma of the Palate: A Case Report. Journal of Clinical and Diagnostic Research. 2012;6(6):1109-1111.

9. Rahnama M, Urszula Orzędała Koszel, Czupkałło L, et al. Pleomorphic Adenoma of the palate: a case report and review of the literature. Wspolczesna Onkol. 2013;17(1):103-106.

10. http://www.springer.com/medicine/surgery/journal/12663

11. Rada R. Obstructive sleep apnea and head and neck neoplasms Otolaryngology-Head and Neck Surgery. 2005;132(5):794-799.

12. Giddings C, Bray D, Rimmer J, et al. Pleomorphic adenoma and severe obstructive sleep apnoea. The Journal of Laryngology \& Otology. 2005;119(3):226-229.

13. Frazell EL. Clinical aspects of tumours of the major salivary glands Cancer. 1954;7(4):637-642.

14. Izzo L, Frati R, Cianfrone F, et al. Parotid gland cancer: a case report of a primitive adenocarcinoma arising from a multirecurrent pleomorphic adenoma. J Exp Clin Cancer Res. 2004;23(1):153-156. 